OPEN ACCESS

Edited by:

Petr Smýkal,

Palacký University, Olomouc, Czechia

Reviewed by:

Hamid Khazaei,

University of Saskatchewan, Canada

Marinus J.M. Smulders,

Wageningen University \& Research,

Netherlands

*Correspondence:

Anete P. de Souza

anete@unicamp.br

tThese authors have contributed equally to this work.

Specialty section:

This article was submitted to

Plant Breeding,

a section of the journal

Frontiers in Plant Science

Received: 18 December 2017 Accepted: 28 May 2018 Published: 03 July 2018

Citation:

de Souza LM, dos Santos LHB,

Rosa JRBF, da Silva CC,

Mantello CC, Conson ARO,

Scaloppi EJ Jr., Fialho JdF

de Moraes MLT, Gonçalves PdS,

Margarido GRA, Garcia AAF,

Le Guen V and de Souza AP (2018)

Linkage Disequilibrium and Population

Structure in Wild and Cultivated

Populations of Rubber Tree (Hevea

brasiliensis). Front. Plant Sci. 9:815.

doi: 10.3389/fpls.2018.00815

\section{Linkage Disequilibrium and Population Structure in Wild and Cultivated Populations of Rubber Tree (Hevea brasiliensis)}

Livia M. de Souza't, Luciano H. B. dos Santos ${ }^{1 \dagger}$, João R. B. F. Rosa ${ }^{2,3}$, Carla C. da Silva ${ }^{1}$, Camila C. Mantello ${ }^{1,4}$, André R. O. Conson ${ }^{1}$, Erivaldo J. Scaloppi Jr. ${ }^{5}$, Josefino de F. Fialho6, Mario Luiz T. de Moraes ${ }^{7}$, Paulo de S. Gonçalves ${ }^{5}$, Gabriel R. A. Margarido², Antonio A. F. Garcia², Vincent Le Guen ${ }^{8}$ and Anete P. de Souza ${ }^{1,9 *}$

${ }^{1}$ Center of Molecular Biology and Genetic Engineering Center, University of Campinas, Campinas, Brazil, ${ }^{2}$ Departamento de Genética, Escola Superior de Agricultura "Luiz de Queiroz" Universidade de São Paulo, Piracicaba, Brazil, ${ }^{3}$ FTS Sementes S.A., Research and Development Center, Ponta Grossa, Brazil, ${ }^{4}$ The John Bingham Laboratory, National Institute of Agricultural Botany, Cambridge, United Kingdom, ${ }^{5}$ Center of Rubber Tree and Agroforestry Systems, Agronomic Institute of Campinas, Votuporanga, Brazil, ${ }^{6}$ Centro de Pesquisa Agropecuária dos Cerrados (Cpac), Embrapa Cerrados, Empresa Brazileira de Pesquisa Agropecuária, Planaltina, Brazil, ${ }^{7}$ Departamento de Fitotecnia, Faculdade de Engenharia de llha Solteira, Universidade Estadual Paulista, Ilha Solteira, Brazil, ${ }^{8}$ Centre de Coopération Internationale en Recherche Agronomique pour le Développement, UMR AGAP, Montpellier, France, ${ }^{9}$ Department of Plant Biology, Biology Institute, University of Campinas, Campinas, Brazil

Among rubber tree species, which belong to the Hevea genus of the Euphorbiaceae family, Hevea brasiliensis (Willd. ex Adr.de Juss.) Muell. Arg. is the main commercial source of natural rubber production worldwide. Knowledge of the population structure and linkage disequilibrium (LD) of this species is essential for the efficient organization and exploitation of genetic resources. Here, we obtained single-nucleotide polymorphisms (SNPs) using a genotyping-by-sequencing (GBS) approach and then employed the SNPs for the following objectives: (i) to identify the positions of SNPS on a genetic map of a segregating mapping population, (ii) to evaluate the population structure of a germplasm collection, and (iii) to detect patterns of LD decay among chromosomes for future genetic association studies in rubber tree. A total of 626 genotypes, including both germplasm accessions (368) and individuals from a genetic mapping population (254), were genotyped. A total of 77,660 and 21,283 SNPs were detected by GBS in the germplasm and mapping populations, respectively. The mapping population, which was previously mapped, was constructed with 1,062 markers, among which only 576 SNPs came from GBS, reducing the average interval between two adjacent markers to $4.4 \mathrm{cM}$. SNPs from GBS genotyping were used for the analysis of genetic structure and LD estimation in the germplasm accessions. Two groups, which largely corresponded to the cultivated and wild populations, were detected using STRUCTURE and via principal coordinate analysis. LD analysis, also using the mapped SNPs, revealed that non-random associations varied along chromosomes, with regions of high LD interspersed with regions of low LD. Considering the length of the genetic map $(4,693 \mathrm{cM})$ and the mean LD $(0.49$ for cultivated and 
0.02 for wild populations), a large number of evenly spaced SNPs would be needed to perform genome-wide association studies in rubber tree, and the wilder the genotypes used, the more difficult the mapping saturation.

Keywords: linkage mapping, genetic diversity, population structure, non-random association, genome-wide association study, Hevea

\section{INTRODUCTION}

Hevea brasiliensis, or the rubber tree, is an important crop species that produces a high-quality natural rubber in commercially viable quantity, accounting for more than $98 \%$ of the total natural rubber production worldwide (Priyadarshan and Goncalves, 2003). A native species of the Amazon rainforest, $H$. brasiliensis is a diploid $(2 n=36, n=18)$, perennial, and cross-pollinated tree species with an estimated haploid genome size of $1.47 \mathrm{~Gb}$ (Tang et al., 2016). This species belongs to the Euphorbiaceae family, comprising 11 inter-crossable species, of which $H$. brasiliensis is the most economically important (Gonçalves et al., 1990). The rubber tree has a heterozygous nature, with a long growing cycle that includes 5 years before latex collection. Like most forest trees, the rubber tree has a long generation time, which explains the slow progress of breeding this species and elucidating the genetic architecture of its complex traits using traditional approaches. Genetic breeding programs are challenged by a low seed yield per pollination (an average of ten seeds obtained for 100 pollinated flowers) and inbreeding depression, making it difficult to develop the appropriate progeny for classical genetic studies (Lespinasse et al., 2000). Hence, relatively little is known about genome-wide models of recombination, allele frequency variation, and linkage disequilibrium (LD) in this important plant.

Over the last 15 years, many genetic maps of the rubber tree have been constructed. The first rubber tree marker-based genetic maps were built with restriction fragment length polymorphisms (RFLPs) and amplified fragment length polymorphisms (AFLPs) (Lespinasse et al., 2000), and dense genetic maps were subsequently constructed using simple sequence repeats (SSRs) (Le Guen et al., 2011; Triwitayakorn et al., 2011; Souza et al., 2013). Saturated genetic linkage maps are important for the identification of genomic regions containing major genes and quantitative trait loci (QTLs) controlling agronomic traits, and such maps are important for further breeding programs.

In recent years, advances in next-generation sequencing technology (NGS) have lowered the cost of DNA sequencing to the point that genotyping-by-sequencing (GBS) (Elshire et al., 2011) is now feasible for high-diversity, large-genome species, and a genetic map has been developed using the GBS approach (Pootakham et al., 2015). GBS utilizes restriction enzymes to capture a reduced representation of the target genome, and with DNA-barcoded adapters, it is possible to sequence multiple samples in parallel in a single run using an NGS platform. GBS has recently been applied to the large barley (Hordeum vulgare L.) and wheat (Triticum aestivum) genomes and has been shown to be an effective tool for developing molecular markers for these species (Poland et al., 2012).
Evaluation of the molecular diversity encompassed in rubber tree genetic resources is a prerequisite for their efficient exploitation in breeding and the development of conservation strategies of genetic diversity. de Souza et al. (2015) analyzed approximately 1,000 cultivated rubber tree accessions originating from various geographic areas in a Brazilian germplasm collection. These accessions were genotyped with 13 SSR markers distributed across the chromosomes of the species, and a total of 408 alleles were identified, 319 of which were shared between groups, while 89 alleles were specific to different groups of accessions.

LD is the non-random association of alleles at distinct loci in the genome of a sampled population (Weir, 1979) and is the basis for association mapping approaches. LD can be used for many purposes in plant genomics research and has received considerable attention as a tool for the study of marker-trait associations due to physical linkage, followed by marker-assisted selection (MAS). Another important application of LD is the study of genetic diversity in natural populations and germplasm collections, where it can be employed for the evaluation of population genetics and in crop improvement programs, respectively (Gupta et al., 2005).

With the rise of sequence-based genotyping, precise and accurate estimates of population structure and the LD across the genome are now attainable for the rubber tree. Our goals in this study were to characterize the genome distribution of single-nucleotide polymorphisms (SNPs) in a rubber tree mapping population using GBS technology, to examine population structure, to investigate how LD breakdown relates to chromosomes, and to compare the LD between cultivated and wild populations. For this study, we used accessions selected from the germplasm collection previously analyzed by de Souza et al. (2015) and a mapping population (PR255 × PB217) previously saturated with SSR markers described by Souza et al. (2013) and Rosa et al. (unpublished).

\section{MATERIALS AND METHODS}

\section{Plant Materials and DNA Extraction}

Two sets of samples were selected for this study, and a total of 626 samples were sequenced. One set consisted of 368 $H$. brasiliensis accessions, composed of both wild germplasm and cultivated genotypes. Details of the plant materials can be found in de Souza et al. (2015) and Supplementary Table S1. The other set is an important mapping population of the rubber tree consisting of 252 F1 hybrids, derived from a cross between PR255 × PB217 and comprising three replicates of each parental genotype, which were mapped with 505 markers (SSRs, expressed sequence tag-SSRs, and SNPs) 
prior to this publication (Souza et al., 2013; Rosa et al., unpublished). Genomic DNA was extracted from leaves using the DNeasy ${ }^{\circledR}$ Plant Mini Kit (QIAGEN, Germany) according to the procedures described by the manufacturer. DNA quality parameters and concentrations were measured using a UV-Vis spectrophotometer (NanoDrop, Thermo Scientific, Wilmington, DE, United States) and agarose gels.

\section{SNP Discovery via GBS}

GBS library preparation and sequencing were performed at the Institute of Genomic Diversity (Cornell University, Ithaca, NY, United States) as described by Elshire et al. (2011). Genome complexity was reduced by digesting individual genomic DNA samples with EcoT22I, a methylation-sensitive restriction enzyme. The resultant fragments from each sample were directly ligated to a pair of enzyme-specific adapters and combined into pools. PCR amplification was carried out to generate the GBS libraries, which were sequenced on the Illumina HiSeq 2500 platform (Illumina Inc., United States). The raw data were processed, and SNP calling was performed using TASSEL 5.0 (Glaubitz et al., 2014). Initially, the FASTQ files were demultiplexed according to the assigned barcode. The reads from each sample were trimmed, and the tags were identified using the following parameters: Kmer length of $64 \mathrm{bp}$, minimum quality score within the barcode and read length of 20, minimum Kmer length of 20. All sequence tags from each sample were aligned to the reference rubber tree genome (Tang et al., 2016) with Bowtie 2 (Langmead and Salzberg, 2012) using the very-sensitive option.

To perform the analysis, the data were divided into the mapping population and germplasms. SNP calling was performed using the TASSEL 5 GBSv2 pipeline (Glaubitz et al., 2014) and filtered using VCFtools (Danecek et al., 2011) with the following criteria: (1) missing data of $20 \%$, (2) minor allele frequency (MAF) greater than or equal to $5 \%$ (MAF 0.05), and (3) biallelic SNPs only.

\section{Genetic Linkage Map}

All linkage analyses were performed using OneMap software (Margarido et al., 2007), version 2.0-1, employing a previously constructed genetic map (Souza et al., 2013; Rosa et al., unpublished) as a basis for the inclusion of GBS-based SNPs with a minimum logarithm of odds (LOD) score of 8.21 (according to the function in the $R$ package Onemap "suggest_lod") and a maximum recombination fraction of 0.35 .

The map construction utilized only markers with 0.05 missing data and tested the pattern of allelic segregation for $\chi^{2}$ goodness of fit to the expected Mendelian segregation ratios, and markers with significant segregation distortion were excluded from further analysis. GBS-based SNPs were added to the previous genetic map using the "try.seq" function in OneMap, which determines the best position for a given unpositioned GBS marker in a specific linkage group (LG). Finally, the fractions of recombination were converted to centimorgans (cM) using the Kosambi map function (Kosambi, 1943), and the map was drawn in Mapchart, version 2.3 (Voorrips, 2002).

\section{Population Structure and Genetic Diversity}

The population structure was investigated in 368 genotypes from the germplasm collection with data from SNPs anchored in a certain LG using two different methods: STRUCTURE analysis and principal coordinate analysis (PCoA). Initially, the structure was analyzed with the software STRUCTURE 2.3.4 (Pritchard et al., 2000). Ten replications were run for each of the subpopulation numbers $(K)$, ranging from 1 to 10 . Each run included 500,000 Markov chain Monte Carlo (MCMC) iterations, among which the first 100,000 iterations (used to monitor whether a chain reached stationarity) were discarded as burn-in. The delta $K$ method was used to identify the number of subgroups in the dataset (Evanno et al., 2005). Based on the posterior probability of membership (Q) of a given accession, the accession was classified as admixed in clusters with a membership of $Q<0.70$. Subsequently, genetic distances between pairs of accessions were calculated, and PCoA was performed for the SNPs using the GenAlEx program (version 6.5) (Peakall and Smouse, 2012).

\section{Analysis of LD}

LD was measured by calculating the squared correlation coefficient $\left(r^{2}\right)$ between each pair of SNPs with the $R$ software and GGT 2.0 (van Berloo, 2008), using data from SNPs anchored in the genetic map. We selected markers that were positioned on the linkage map and were present in the germplasm to calculate the LD in each LG separately, considering the subgroup inferred with STRUCTURE. The decay of LD over genetic distance was investigated by plotting pair-wise $r^{2}$ values against the distance (cM) between markers on the same chromosome using the following model: $y=a+b^{-c / x}$ (Ranc et al., 2012), where $x$ and $y$ represent the genetic distance in $\mathrm{cM}$ and the estimated $r^{2}$, respectively. The critical $r^{2}$ for LD decay was determined by values of 0.1 , which is considered the minimum threshold for a significant association between pairs of loci and to describe the maximum genetic or physical distance at which LD is significant (Zhu et al., 2008).

\section{RESULTS}

\section{SNP Discovery and Evaluation}

The analysis was performed separately for the mapping population and germplasm to produce a total of 1.785 million reads of sequence data, of which $89 \%$ (1,586 million reads) consisted of good barcoded reads. Of these reads, 69,408 (23.42\%) were aligned to the mapping population exactly one time and $177,143(59.78 \%)$ more than one time, corresponding to an $83.20 \%(246,551)$ overall alignment rate. The total rate of alignment to the germplasm was $89.97 \%(818,807)$, of which $22.59 \%$ of the tags $(205,576)$ were aligned to the rubber tree reference genome exactly one time (Tang et al., 2016) and 67.38\% $(613,231)$ more than one time.

A total of 386,180 SNPs were identified in the germplasm, and 76,191 SNPs were detected in the mapping population, of 
which 350,965 SNPs (germplasm) and 66,453 SNPs (mapping population) were biallelic. After excluding markers showing (1) more than $20 \%$ missing data or (2) a MAF $\leq 0.05$, the whole dataset was reduced to 77,660 and 21,283 SNPs in the germplasm and mapping populations, respectively. The SNP frequencies were one biallelic SNP every $20.7 \mathrm{~kb}$ for the mapping population and every $3.9 \mathrm{~kb}$ for the germplasm.

Sequence data are deposited under EMBL-EBI accession PRJEB26962.

\section{Saturation of the Linkage Map With SNPs}

From 21,283 SNPs, a total of 14,852 markers were selected after applying the chi-square test $(P \leq 0.05)$, which revealed segregation ratios of 1:2:1 and 1:1. Markers exhibiting statistically significant segregation distortion were excluded from further analysis to obtain accurate genetic linkage maps. The data analyses were performed using SNPs with a maximum of $5 \%$ missing data, resulting in a linkage map with 1,062 markers presenting 348 SSR markers (Souza et al., 2013; Mantello, 2014), 576 SNPs from GBS (Supplementary Table S2) and 138 SNPs markers genotyped using the Sequenom MassARRAY ${ }^{\circledR}$ platform (AgenaBio, San Diego, CA, United States) and the Fluidigm ${ }^{\circledR}$ platform (South San Francisco, CA, United States), developed from de novo transcriptome assemblies (Mantello et al., 2014; Salgado et al., 2014) and from EST full-length libraries (Silva et al., 2014).

The genetic map was organized according to the numbers obtained from the map previously developed by Rosa et al. (unpublished). Eleven markers were removed after data diagnosis using heat map graphs, thereby permitting the visualization of the recombination fraction and LOD scores from markers, to group the SNPs in the LGs without changing the order of the base map. Only LG18 was further divided into subgroups "A" and "B" (Figure 1).

Thus, a genetic map was generated spanning a cumulative length of 4,693 cM (Figure 1), distributed among all the chromosomes. The LGs ranged from $23.8 \mathrm{cM}$ (LG18B) (Supplementary Table S3) with 14 markers to the largest group with $404.6 \mathrm{cM}$ (LG10) with 85 markers (of which 78 were from GBS), and the average interval size between two adjacent markers was $4.4 \mathrm{cM}$.

The maximum gap size was $36.4 \mathrm{cM}$ (LG11), which was maintained from the previous map. Furthermore, some regions could not be sampled using the selected restriction enzyme; in LG16, for example, only two GBS-based markers were added.

\section{Genetic Relationships Among Populations}

The germplasm collection was selected in a prior work by de Souza et al. (2015) examining the population structure with SSR markers. To confirm the population structure of the sample of selected individuals, we performed a new analysis as performed with the SNPs from GBS genotyping. Only mapped SNPs that were common in the germplasm collection were used. Clustering inference performed with $K$ values ranging from 1 to 10 showed that the model likelihood increased steeply at $K=2$, followed by a drastic decline starting at $K=3$, suggesting that the optimal $K$ value was 2 . The assignment results for $K=2$ showed that some of the sampled individuals exhibited admixtures from two gene pools (Figure 2A): group 1 (red bars) mainly consisted of accessions originating from the Mato Grosso and cultivated genotypes, and group 2 (green bars) consisted entirely of wild accessions from Amazonas, Rondônia, Para, and Acre (identified as IS - Ilha Solteira). Twenty-one accessions showing a membership probability ( $Q$ value) below 0.70 were defined as admixed and were removed from subsequent analyses.

As a second analysis of differentiation, we employed PCoA based on a similarity matrix that explained 22.8 and $16.7 \%$ of the genetic variation with the first and second PCoA axes, respectively. Plotting the two first PCoA axes separated the germplasm into two clusters, though some overlap was present between the wild germplasms and cultivated genotypes (Figure 2). On the first axis, most of the breeding genotypes were separated from the other genotypes. On the second axis, the wild germplasm samples were clustered together, but some accessions from the Amazon were isolated in their own subdivision.

\section{Evaluation of LD}

Based on population genetic structure, accessions could be divided into two distinct groups (cultivated and wild group) (Figure 2), and pairwise LD estimates were performed within the gene pool of each of these groups. To visualize LD throughout the genome, heat maps were produced based on pairwise $r^{2}$ estimates, and corresponding $p$-values were calculated using permutations for all marker pairs (Supplementary Figure S1). These heat maps were employed to identify variations in LD between the cultivated and wild rubber tree germplasm groups.

In 16,025 pairwise combinations, we identified $186(1.2 \%)$ and $592(3.7 \%)$ statistically significant associations that were in LD $(P<0.05)$ in the cultivated and wild germplasms, respectively. Of these significant associations, 78 and 64 were intrachromosomal in the cultivated and wild germplasms, respectively, accounting for 0.5 and $0.4 \%$ of the total possible intrachromosomal correlations. Among the unlinked loci, the proportions of LD were 0.7 and $3.3 \%$ for the cultivated and wild germplasms, respectively. In both clusters, an uneven distribution of LD among the 18 chromosomes was observed.

The strength of LD $(P<0.05)$ was very different between two clusters, as reflected by the mean $r^{2}$ values of 0.49 and 0.02 obtained for the cultivated and wild germplasms, respectively. The 346 SNPs that were localized in the integrated map were used for the estimation of LD decay among the different LGs. LD decay was estimated across the germplasms and was found to be more pronounced in the wild germplasms, with the range being dependent on the chromosome group (Supplementary Figure S2). Using a fixed baseline $r^{2}$ value of 0.1 for the wild germplasm, LD decay ranged from $2.3 \mathrm{cM}$ (LG9) to $11.4 \mathrm{cM}$ (LG17). In contrast, in the cultivated accessions, this decay was slower, ranging from $4.3 \mathrm{cM}$ (LG8) to $50 \mathrm{cM}$ (LG17), with average values of $5.6 \mathrm{cM}$ and $19.6 \mathrm{cM}$ for the wild and cultivated germplasms, respectively (Figure 3). The patterns of LD can also be visualized across the genome from the diagonal of the heat maps (Supplementary Figure S1). 


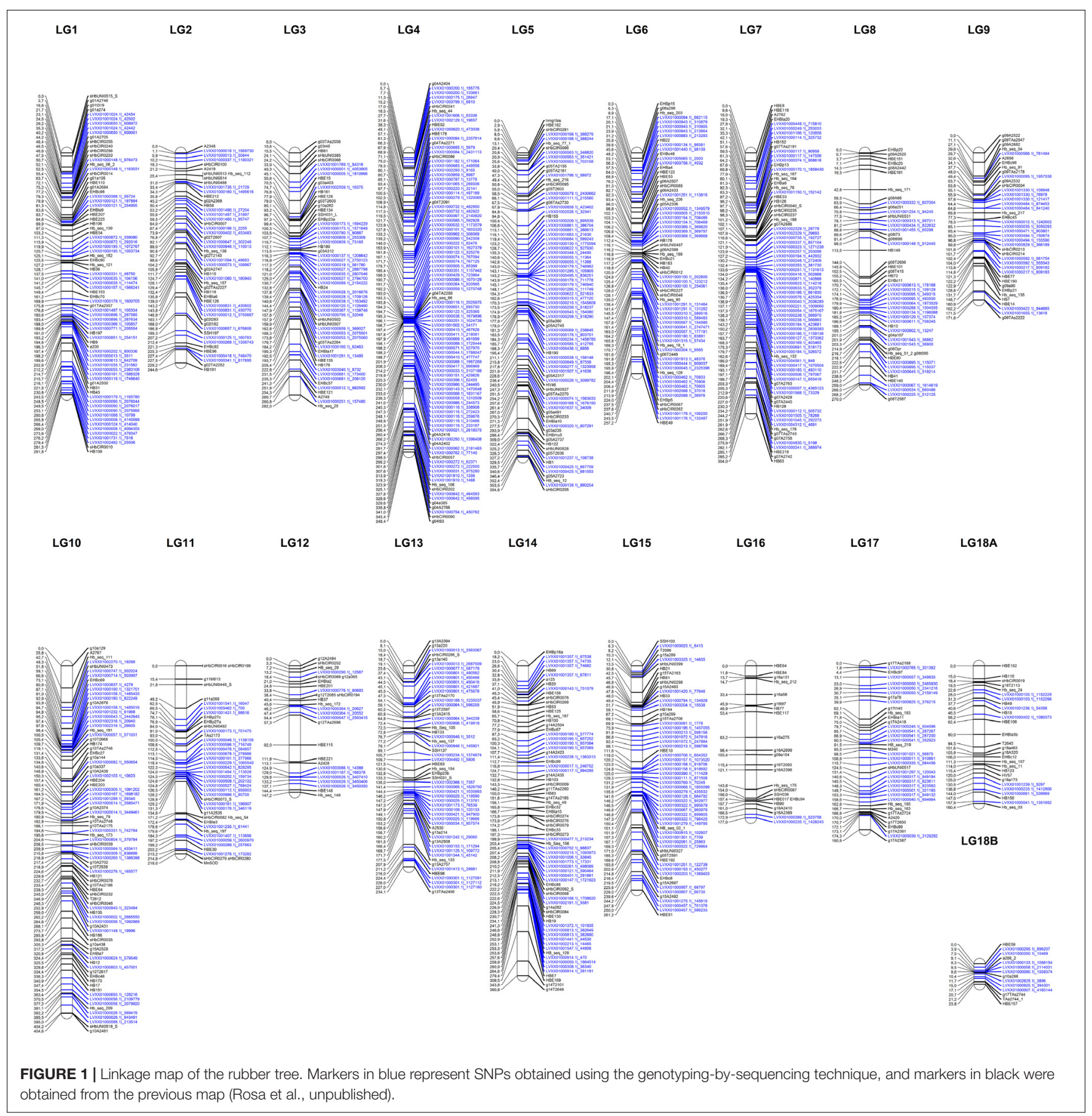

\section{DISCUSSION}

We performed a genetic mapping study with a bi-parental population (252 segregating hybrids between PR255 × PB217) and analyzed the population structure and LD with two different populations of rubber tree, formed from 47 accessions from a breeding program and 300 accessions from a germplasm collection. For this study, we employed SNPs obtained via the GBS approach, which enabled the detection of polymorphisms distributed across the genome.
An important breakthrough of the GBS approach is that a reference genome is not necessary for SNP genotyping. However, the availability of a reference genome offers additional benefits, as it allows proper alignment and ordering of the sequenced tags (Poland et al., 2012). The first draft of the genome sequence (Rahman et al., 2013) provided a source of genomic information, after which three more genomes were published (Lau et al., 2016; Tang et al., 2016; Pootakham et al., 2017). The assembly is highly fragmented, containing more than 1 million contigs. The assembly of a complex 

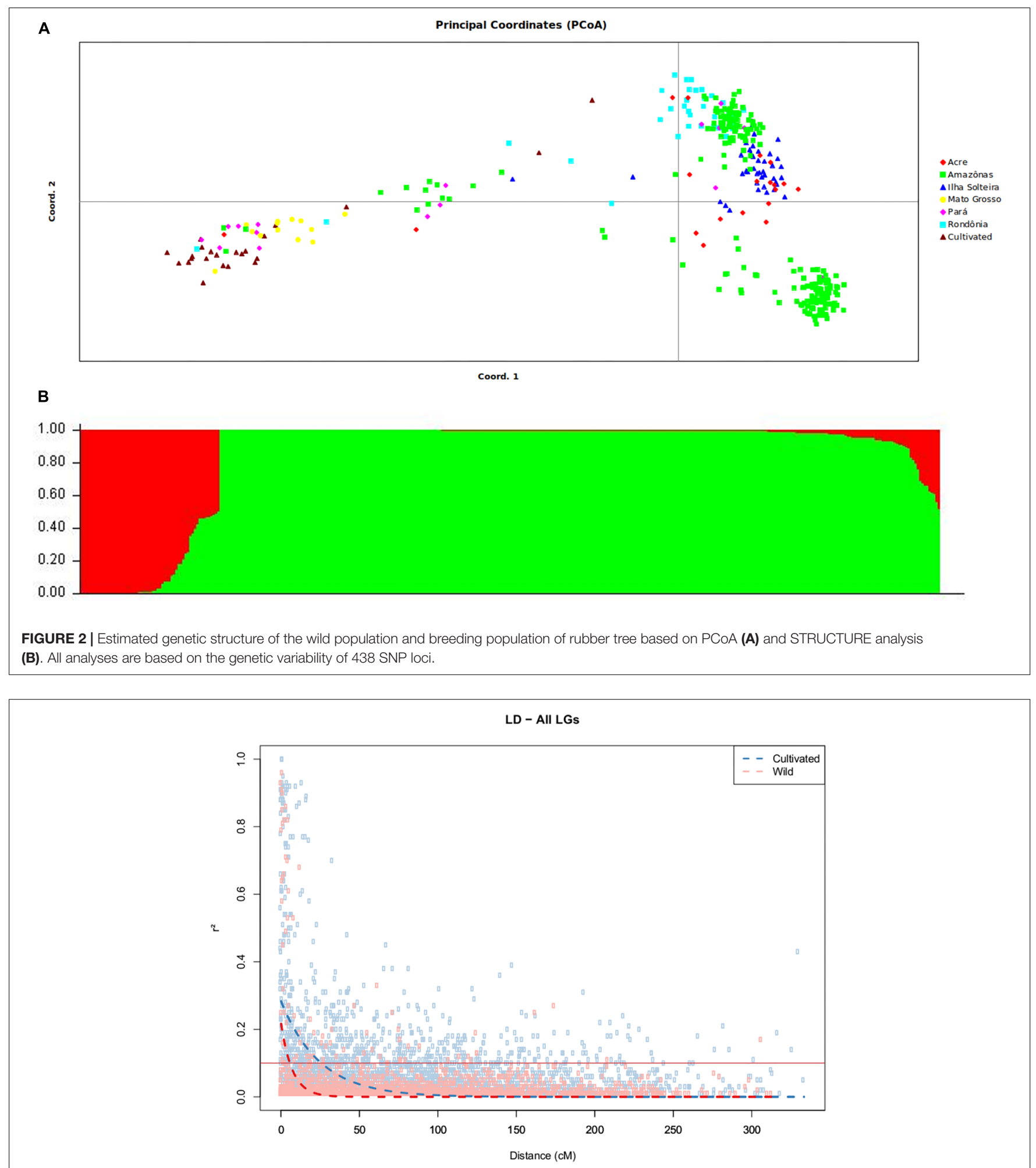

FIGURE 3 | Decay of LD $\left(r^{2}\right)$ as a function of genetic distance (cM) between pairs of loci on all chromosomes. Only $r^{2}$ values with $P<0.05$ are shown.

genome is challenging, owing in part to the presence of highly repetitive DNA sequences, which introduce ambiguity during genome reconstruction. We used the genome published by
Tang et al. (2016) to map the SNPs. The SNPs positioned on the map are named in reference to the location of alignment with the genome. 
Repetitive regions account for $71 \%$ of the Hevea genome (Tang et al., 2016), posing a major challenge for the de novo assembly, particularly when exclusively short-read data are used. This phenomenon might explain the prevalence of tags that aligned to more than one unique region (59.78\% to the mapping population and $67.38 \%$ to the germplasm) (Tang et al., 2016). Based on the properties of the reference genome (characterized by a great accumulation of repetitive sequences, primarily in heterochromatic regions) (Rahman et al., 2013), the restriction enzyme EcoT22I was selected because it is partially sensitive to methylation and rarely cuts retrotransposons.

The frequency of nucleotide substitutions was five times higher in the wild $(3.9 \mathrm{~kb})$ than in the cultivated $(20.7 \mathrm{~kb})$ germplasm sequences. One explanation for this finding is that selection in cultivated breeding programs acts to reduce diversity and alter allele frequencies in the DNA sequence. Depending on how LD is increased surrounding these loci; the effects of such a selection may not extend sufficiently far to affect the overall genome diversity. Rubber tree breeders have had to develop cultivars that are appropriate for the specific temperature and humidity conditions encountered in different cultivation areas, along with various biotic and abiotic stress resistance factors. High-density SNPs are also common in non-genetic regions where there is no selection pressure, and the abundance of these SNPs will be very useful for future assessments of breeding populations. The high diversity of SNPs is characteristic of outbreeding trees; in other studies examining rubber tree, Pootakham et al. (2011) previously identified a frequency of one SNP every $1.5 \mathrm{~kb}$, while Pootakham et al. (2015) observed a frequency of one SNP every 308 nucleotides.

GBS does not require complete genome sequencing; only a targeted sequencing approach is necessary. Due to its highthroughput efficiency, GBS has been used for SNP identification and mapping in many plant species (Poland et al., 2012; Rabbi et al., 2014; Bekele et al., 2018; Rimbert et al., 2018). In rubber tree, most of the genetic linkage maps constructed to date have employed molecular markers such as RFLPs and AFLPs (Lespinasse et al., 2000) or microsatellites (SSRs) (Le Guen et al., 2011; Triwitayakorn et al., 2011; Souza et al., 2013). However, GBS was employed for linkage map construction in rubber tree very recently (Pootakham et al., 2015; Shearman et al., 2015). In the present study, we utilized the GBS platform to sequence a PR255 $\times$ PB217 mapping population that was previously saturated with SSR markers and SNPs obtained from other specific platforms (Souza et al., 2013; Rosa et al., unpublished).

The linkage map constructed in the present study exhibits a regular marker distribution. However, the cumulative genetic map is significantly longer $(4,693 \mathrm{cM})$ than would be expected for maps derived from other mapping populations that have presented a lower resolution; for example, a map of 2,052 cM (Pootakham et al., 2015) was obtained using only SNPs, and a map of 2,441 cM (Le Guen et al., 2011) was obtained using both SSRs and AFLPs. Such expansions of the rubber tree genetic linkage map have also reported previously $(4,160 \mathrm{cM}$, Shearman et al., 2015). Several factors may be responsible for this phenomenon, including the numbers and types of mapped loci, the genetic constitution of different mapping populations and differences in mapping strategies, the mapping software and the ratio between the number of markers and the population size (Knox and Ellis, 2002).

Genetic mapping of the population (PR255 × PB217) was initiated in the first publication by Souza et al. (2013) using microsatellite markers. These first LGs were organized according to the numbers obtained from the map previously developed by Lespinasse et al. (2000), and information from other maps (unpublished) consisting of microsatellites in common was used to identify syntenic markers. Subsequently, the PR255 $\times$ PB217 mapping population was saturated with SSR markers and 243 SNPs obtained from platforms such as Sequenom MassARRAY iPLEX technology and KASP genotyping (Rosa et al., unpublished). The SNPs from GBS used to construct the new genetic linkage map contributed to reducing the average interval between two adjacent markers ( $4.4 \mathrm{cM}$ versus $7.4 \mathrm{cM}$ ). However, the marker density remained lower than that of the first rubber tree map (Lespinasse et al., 2000), which presented one marker every 3 or $0.89 \mathrm{cM}$ according to Pootakham et al. (2015). LG11 displayed the maximum gap size (36.4 cM), which may have been caused by sections of the genome that were identical among the parental genotypes and thus an absence of polymorphisms, or by recombination hotspots. Large gaps exhibiting a low degree of polymorphisms have also been reported by Shearman et al. (2015).

Genetic maps are important tools not only for QTL mapping but also for anchoring genome assembly scaffolds into pseudochromosomes. The draft genome of $H$. brasiliensis has been reported to be highly heterozygous, with $71 \%$ of the genome length comprising repeats (Tang et al., 2016). A total of 21,283 biallelic SNPs were identified, potentially aligning with 1,926 scaffolds, corresponding to $26 \%$ of the total scaffolds of the genome used. However, only $3 \%$ of these scaffolds were anchored to LGs due to the relatively fragmented scaffold sequences and the usage of an insufficient genetic map to anchor the scaffolds. Thus, it is important to develop higher-quality genetic maps to help improve the scaffold anchoring ratio of future rubber tree genome assemblies.

Progress in the development of new molecular markers and genetic linkage maps is important for genetic improvement in rubber tree breeding programs. Despite the economic and ecological importance of this crop, restricted genomic resources are available for rubber tree. Sequencing of the studied family allowed the identification and genotyping of many markers in an efficient and cost-effective way. The linkage mapping analysis resulted in a number of LGs corresponding to the rubber tree haploid chromosome number $(n=18)$ (Ong, 1975; Lespinasse et al., 2000), and the development of GBS methods and genetic maps represents an important advancement of the genomics tools available for these crops, which currently lack a good reference genome sequence.

Diversity analyses were performed in a previous work that included the entire collection of rubber germplasm, analyzing 1,117 genotypes with 13 microsatellite markers (de Souza et al., 2015), and showed a mean observed heterozygosity (Ho) of 0.64 and higher genetic diversity $(\mathrm{He})$ than $\mathrm{Ho}$ in all cases. Wright's fixation index $(\mathrm{F})$ values were positive, with a mean of 
0.16 obtained for the accessions overall. Among a total of 408 observed alleles, 89 represented unique alleles to different groups of accessions, demonstrating the high level of heterogeneity in these genotypes. To confirm the population structure of the sample of selected individuals based on the work of de Souza et al. (2015), a new analysis of the population structure was conducted with SNPs obtained from GBS genotyping. The $\Delta \mathrm{K}$ values obtained in this study indicated that the rubber tree germplasm could be divided into two groups and showed that some of the sampled individuals exhibited admixtures from two gene pools, which was also confirmed by plotting the two first PCoA axes.

Recent work using SSR markers has revealed similar results to those obtained using SNPs from GBS. For example, Le Guen et al. (2009) demonstrated a separation between the Acre and Rondônia groups and the Mato Grosso group. One possible reason for this separation is that the wild accessions are from geographically distant populations that are not connected by hydrographic networks (Le Guen et al., 2009; Chanroj et al., 2017), whereas most of the Wickham clones were collected from regions that are geographically closer to the Mato Grosso and the Tapajós river, enabling rubber seeds to flow from one region to another via the river. de Souza et al. (2015) reported a difference in genotypes between the two groups, which are in different river basins, thus separating the genotypes in the cultivated and wild groups. Within the group of cultivated genotypes denoted as Mato Grosso and Wickhan, genotypes collected from Mato Grosso were genetically close to genotypes used to initiate the Asian breeding programs, denoted Wickhan genotypes in this article. These genotypes were collected by Henry Wickhan in 1976 in the same basin from which the genotypes from Mato Grosso were sampled (Gonçalves et al., 1990). The results obtained by de Souza et al. (2015) corroborate the seminal study by Le Guen et al. (2009).

Population genetic structure is a principal factor influencing the generation of false positives and the success of the association or LD mapping (Gupta et al., 2005). These 368 rubber tree accessions could be divided into two distinct groups based on population genetic structure: cultivated and wild germplasm. The investigation of LD decay versus genetic distance based on markers is not possible without prior information regarding the positions of markers in the genome. Since the information on the rubber tree genome is still inaccurate regarding these positions, we employed the existing genetic map of the PR255 $\times$ PB217 population to identify the positions of some SNPs and, thus, enable a more accurate study of LD decay.

LD analysis with the mapped SNPs revealed that LD varied along the chromosomes, with regions of high LD being interspersed with regions of low LD (Supplementary Figure S1). LD estimation is possible without the positions of molecular markers along the genome and was indeed performed in this study. However, these positions are crucial if one is interested in determining the decay or extension of $\mathrm{LD}$ in relation to the genetic distance, regardless of by chromosome.

The average $r^{2}$ values were found to be very different when the two detected groups were compared. This measurement was higher in the breeding germplasm (0.49) than in the wild germplasm (0.02), corroborating the results of Chanroj et al. (2017), who suggested that these results were caused by high gene flow in the wild Amazonian population. The breeding accessions exhibited a notably higher level of LD, suggesting less genetic diversity in this subdivision, perhaps because of the constraint of genetic variability employed in breeding programs due to the recurrent process of selection. The low LD detected in the wild germplasm in this study was expected because perennial outcrossing tree species display a high effective recombination rate, which leads to the rapid decay of LD (Krutovsky and Neale, 2005).

The higher LD level detected in the cultivated group may have been influenced by the partly identical-by-descent of these genotypes from a limited number of founders of the breeding programs, with only a few generations between them. Thus, many longer pieces of chromosomes have not had time to undergo disruptions. Domesticated crop cultivars necessarily represent a subset of the genetic variation found in their wild ancestors, and the process of crop domestication is responsible for genetic bottlenecks (McCouch, 2004). Although rubber tree breeding programs are very recent, the differences in LD patterns detected between cultivated and wild germplasms suggest that plant breeders may have selected for separate combinations of genes during the breeding process. Moreover, selection for high latex yields and the extensive use of particular clones as parents in rubber breeding programs have further reduced the genetic diversity of commercial rubber germplasm (Priyadarshan, 2016), which may also affect LD.

LD decay was estimated at $25.7 \mathrm{cM}$ within cultivated and $5.7 \mathrm{cM}$ within wild germplasm, and significant interchromosomal LD was identified within cultivated in contrast to wild germplasm. The distances of LD decay between the two groups were most different in LGs 3, 5, 7, 10, and 11, suggesting that these chromosomes may carry more genes related to agronomic traits that have been selected via organized breeding of this crop. In previous studies involving Amazonian accessions of rubber tree, Chanroj et al. (2017) revealed an LD decay of more than $0.5-6 \mathrm{cM}$ and suggested that LD estimates were significantly influenced by physical distance, with LD decay greater than $2 \mathrm{kbp}$ being observed in the widespread Amazonian population. These authors showed that LD decay over genetic distance was different for the 18 different chromosomes, possibly because of the different recombination rates of the 18 chromosomes. Rapid LD decay has been reported for many other outcrossing tree species, such as Populus nigra, in which a decay of $r^{2}$ with distance in the CAD4 gene was observed at approximately 16 bp (Marroni et al., 2011). In Eucalyptus globulus, candidate genes for wood quality were analyzed using SNPs, and LD was estimated to decay rapidly (Thavamanikumar et al., 2011). Most LD estimation studies conducted in tree species are based on candidate genes (Krutovsky and Neale, 2005).

LD mapping relies on germplasm samples and, as such, does not require the development of experimental crosses with specific genetic backgrounds, and thus ease of use is an obvious 
benefit in studies of perennial species with long life cycles. $\mathrm{LD}$ is a key factor in determining the number of markers needed for genome-wide association studies (GWAS) and genomic selection (GS). Genomes with low LD require a high marker density for GWAS or GS; therefore, our SNPs may be valuable for GWAS in rubber tree breeding. Considering the length of the genetic map $(4,693 \mathrm{cM})$ and the mean LD observed ( 0.49 in breeding and 0.02 in wild populations), many evenly spaced SNPs would be necessary to perform GWAS in the rubber tree, and the wilder the genotypes that are used, the more difficult is the saturation of the mapping. However, to obtain a sufficient SNP density throughout the genome and to account for variation in LD along the chromosomes more markers must be genotyped. Our study results provide a valuable resource for further genetic studies involving linkage or association mapping, markerassisted breeding and Hevea sequence assembly and comparative mapping.

Furthermore, GWAS of wild germplasm accessions in the future will provide a substantial contribution to dealing with new challenging situations that will arise as a consequence of global climatic changes. Useful QTLs and genes to face these new situations are currently unknown, but the best way to identify them would be through analyses relying on GWAS of large panels of wild genotypes. A precise assessment of the LD pattern across the genome of Hevea is necessary for such endeavors, and the present study supplies a major contribution to this goal.

\section{AUTHOR CONTRIBUTIONS}

LMdS, LHBdS, VLG, and AdS designed the study and performed the experiments. LMdS, LHBdS, AC, CdS, CM, ES, JF, and MdM performed the experiments. LMdS, LHBdS, JR, GM, CM, VLG, MdM, PG, and AG analyzed the data. LMdS, LHBdS, and AdS wrote the manuscript.

\section{REFERENCES}

Bekele, W. A., Wight, C. P., Chao, S., Howarth, C. J., and Tinker, N. A. (2018). Haplotype based genotyping-by-sequencing in oat genome research. Plant Biotechnol. J. doi: 10.1111/pbi.12888 [Epub ahead of print].

Chanroj, V., Rattanawong, R., Phumichai, T., Tangphatsornruang, S., and Ukoskit, K. (2017). Genome-wide association mapping of latex yield and girth in Amazonian accessions of Hevea brasiliensis grown in a suboptimal climate zone. Genomics 109, 475-484. doi: 10.1016/j.ygeno.2017. 1007.1005

Danecek, P., Auton, A., Abecasis, G., Albers, C. A., Banks, E., DePristo, M. A., et al. (2011). The variant call format and VCFtools. Bioinformatics 27, 2156-2158. doi: 10.1093/bioinformatics/btr330 1

de Souza, L. M., Le Guen, V., Cerqueira-Silva, C. B. M., Silva, C. C., Mantello, C. C., Conson, A. R. O., et al. (2015). Genetic diversity strategy for the management and use of rubber genetic resources: more than 1,000 wild and cultivated accessions in a 100-genotype core collection. PLoS One 10:e0134607. doi: 10.1371/journal.pone.0134607

Elshire, R. J., Glaubitz, J. C., Sun, Q., Poland, J. A., Kawamoto, K., Buckler, E. S., et al. (2011). A robust, simple genotyping-by-sequencing (GBS) approach for high diversity species. PLoS One 6:e19379. doi: 10.1371/journal.pone. 0019379

\section{FUNDING}

The authors gratefully acknowledge the Fundação de Amparo a Pesquisa do Estado de São Paulo (FAPESP) (2007/50392-1; 2012/50491-8) for financial support and for graduate scholarships to CM (2011/50188-0 and 2014/18755-0) and CdS (2009/52975-0) and LHBdS (2014/11807-5 and 2017/07908-9) and a post-doctoral fellowship to LMdS (2012/05473-1); the Conselho Nacional de Desenvolvimento Científico e Tecnológico (CNPq) for financial support (478701/2012-8; 402954/2012-2), a post-doctoral fellowship to $\mathrm{CdS}$, and research fellowships to AG, AdS, and PG and, a Ph.D. fellowship to AC; and the Coordenação de Aperfeiçoamento do Pessoal de Nível Superior (CAPES) for financial support (Computational Biology Program and CAPES-Agropolis Program) and post-doctoral fellowships to LMdS and AC.

\section{SUPPLEMENTARY MATERIAL}

The Supplementary Material for this article can be found online at: https://www.frontiersin.org/articles/10.3389/fpls.2018.00815/ full\#supplementary-material

FIGURE S1 | Plots of LD heat maps for (A) with 47 breeding accessions and (B) with 300 accessions from wild germplasms. The rubber tree LGs are represented by a diagonal bar. Markers were ordered on the $x$ - and $y$-axes based on genomic location; therefore, each cell of the heat map represents a single marker pair. The $r^{2}$ values for each marker pair are presented in the bottom half of the heat map and are represented by shades of red increasing in intensity in equal increments of 0.1 from 0.0 (white) to 1.0 (red).

FIGURE S2 | Decay of LD $\left(r^{2}\right)$ as a function of genetic distance (cM) between pairs of loci in individual LGs. Only $r^{2}$ values with $P<0.05$ are shown.

TABLE S1 | Origin of germplasm genotypes and population structure results.

TABLE S2 I SNPS from GBS and their genome information. The name of markers are in agreement with the sequences of the reference genome (Tang et al., 2016).

TABLE S3 | Marker information for the genetic map.

Evanno, G., Regnaut, S., and Goudet, J. (2005). Detecting the number of clusters of individuals using the software STRUCTURE: a simulation study. Mol. Ecol. 14, 2611-2620. doi: 10.1111/j.1365-294X.2005.02553.x

Glaubitz, J. C., Casstevens, T. M., Lu, F., Harriman, J., Elshire, R. J., Sun, Q., et al. (2014). TASSEL-GBS: a high capacity genotyping by sequencing analysis pipeline. PLoS One 9:e90346. doi: 10.1371/journal.pone.0090346

Gonçalves, P. S., Cardoso, M., and Ortolani, A. A. (1990). Origem, variabilidade e domesticação da Hevea: uma revisão. Pesqui. Agropecu. Bras. 25, 135-156.

Gupta, P. K., Rustgi, S., and Kulwal, P. L. (2005). Linkage disequilibrium and association studies in higher plants: present status and future prospects. Plant Mol. Biol. 57, 461-485. doi: 10.1007/s11103-005-0257-z

Knox, M. R., and Ellis, T. H. N. (2002). Excess heterozygosity contributes to genetic map expansion in pea recombinant inbred populations. Genetics 162, 861-873.

Kosambi, D. D. (1943). The estimation of map distances from recombination values. Ann. Eugen. 12, 172-175. doi: 10.1111/j.1469-1809.1943.tb02 321.x

Krutovsky, K. V., and Neale, D. B. (2005). Nucleotide diversity and linkage disequilibrium in cold-hardiness- and wood quality-related candidate genes in Douglas fir. Genetics 171, 2029-2041. doi: 10.1534/genetics.105.04 4420

Langmead, B., and Salzberg, S. L. (2012). Fast gapped-read alignment with Bowtie 2. Nat. Methods 9, 357-359. doi: 10.1038/nmeth.1923 
Lau, N.-S., Makita, Y., Kawashima, M., Taylor, T. D., Kondo, S., Othman, A. S., et al. (2016). The rubber tree genome shows expansion of gene family associated with rubber biosynthesis. Sci. Rep. 6:28594. doi: 10.1038/srep28594

Le Guen, V., Doaré, F., Weber, C., and Seguin, M. (2009). Genetic structure of Amazonian populations of Hevea brasiliensis is shaped by hydrographical network and isolation by distance. Tree Genet. Genomes 5, 673-683. doi: 10.1007/s11295-009-0218-9

Le Guen, V., Garcia, D., Doaré, F., Mattos, C. R. R., Condina, V., Couturier, C., et al. (2011). A rubber tree's durable resistance to Microcyclus ulei is conferred by a qualitative gene and a major quantitative resistance factor. Tree Genet. Genomes 7, 877-889. doi: 10.1007/s11295-011-0381-7

Lespinasse, D., Rodier-Goud, M., Grivet, L., Leconte, A., Legnate, H., and Seguin, M. (2000). A saturated genetic linkage map of rubber tree (Hevea spp.) based on RFLP, AFLP, microsatellite, and isozyme markers. Theor. Appl. Genet. 100, 127-138. doi: 10.1007/s001220050018

Mantello, C. C. (2014). Mapeamento Genético Molecular em Hevea brasiliensis. Ph.D. thesis, Universidade Estadual de Campinas, Campinas.

Mantello, C. C., Cardoso-Silva, C. B., da Silva, C. C., de Souza, L. M., Scaloppi Junior, E. J., de Souza, G. P., et al. (2014). De novo assembly and transcriptome analysis of the rubber tree (Hevea brasiliensis) and SNP markers development for rubber biosynthesis pathways. PLoS One 9:e102665. doi: 10.1371/journal. pone. 0102665

Margarido, G. R., Souza, A. P., and Garcia, A. A. (2007). OneMap: software for genetic mapping in outcrossing species. Hereditas 144, 78-79. doi: 10.1111/j. 2007.0018-0661.02000.x

Marroni, F., Pinosio, S., Zaina, G., Fogolari, F., Felice, N., Cattonaro, F., et al. (2011). Nucleotide diversity and linkage disequilibrium in Populus nigra cinnamyl alcohol dehydrogenase CAD4) gene. Tree Genet. Genomes 7, 1011-1023. doi: 10.1007/s11295-011-0391-5

McCouch, S. (2004). Diversifying selection in plant breeding. PLoS Biol. 2:e347. doi: 10.1371/journal.pbio.0020347

Ong, S. H. (1975). "Chromosome morphology at the pachytene stage in Hevea brasiliensis - a preliminary report," in Proceedings of the International Rubber Conference, Vol. 2 (Kuala Lumpur: IRRDB), 3-12.

Peakall, R., and Smouse, P. E. (2012). GenAlEx 6.5: genetic analysis in Excel. Population genetic software for teaching and research-an update. Bioinformatics 28, 2537-2539. doi: 10.1093/bioinformatics/bts460

Poland, J. A., Brown, P. J., Sorrells, M. E., and Jannink, J. L. (2012). Development of high-density genetic maps for barley and wheat using a novel two-enzyme genotyping-by-sequencing approach. PLoS One 7:e32253. doi: 10.1371/journal. pone. 0032253

Pootakham, W., Chanprasert, J., Jomchai, N., Sangsrakru, D., Yoocha, T., Therawattanasuk, K., et al. (2011). Single nucleotide polymorphism marker development in the rubber tree, Hevea brasiliensis (Euphorbiaceae). Am. J. Bot. 98, e337-e338. doi: 10.3732/ajb.1100228

Pootakham, W., Ruang-Areerate, P., Jomchai, N., Sonthirod, C., Sangsrakru, D., Yoocha, T., et al. (2015). Construction of a high-density integrated genetic linkage map of rubber tree (Hevea brasiliensis) using genotyping-by-sequencing (GBS). Front. Plant Sci. 6:367. doi: 10.3389/fpls.2015.00367

Pootakham, W., Sonthirod, C., Naktang, C., Ruang-Areerate, P., Yoocha, T., Sangsrakru, D., et al. (2017). De novo hybrid assembly of the rubber tree genome reveals evidence of paleotetraploidy in Hevea species. Sci. Rep. 7:41457. doi: $10.1038 /$ srep41457

Pritchard, J. K., Stephens, M., and Donnelly, P. (2000). Inference of population structure using multilocus genotype data. Genetics 155, 945-959.

Priyadarshan, P. M. (2016). "Genetic diversity and erosion in Hevea rubber," in Genetic Diversity and Erosion in Plants: Case Histories, eds M. R. Ahuja and S. M. Jain (Cham: Springer International Publishing), 233-267. doi: 10.1007/ 978-3-319-25954-3_6

Priyadarshan, P. M., and Goncalves, P. S. (2003). Hevea gene pool for breeding. Genet. Resour. Crop Evol. 50, 101-114. doi: 10.1023/A:1022972320696

Rabbi, I. Y., Hamblin, M. T., Kumar, P. L., Gedil, M. A., Ikpan, A. S., Jannink, J. L., et al. (2014). High-resolution mapping of resistance to cassava mosaic geminiviruses in cassava using genotyping-by-sequencing and its implications for breeding. Virus Res. 186, 87-96. doi: 10.1016/j.virusres.2013.12.028
Rahman, A. Y., Usharraj, A. O., Misra, B. B., Thottathil, G. P., Jayasekaran, K., Feng, Y., et al. (2013). Draft genome sequence of the rubber tree Hevea brasiliensis. BMC Genomics 14:75. doi: 10.1186/1471-2164-14-75

Ranc, N., Muños, S., Xu, J., Le Paslier, M. C., Chauveau, A., Bounon, R., et al. (2012). Genome-wide association mapping in tomato (Solanum lycopersicum) is possible using genome admixture of Solanum lycopersicum var. cerasiforme. G3 2, 853-864. doi: 10.1534/g3.112.002667

Rimbert, H., Darrier, B., Navarro, J., Kitt, J., Choulet, F., Leveugle, M., et al. (2018). High throughput SNP discovery and genotyping in hexaploid wheat. PLoS One 13:e0186329. doi: 10.1371/journal.pone.0186329

Rosa, J. R. B., Mantello, C. C., Garcia, D., Souza, L. M., Silva, C. C., Gazaffi, R., et al. (Submitted). QTL detection for growth and latex production in a full-sib population of rubber tree cultivated under suboptimal climate conditions.

Salgado, L., Koop, D., Pinheiro, D., Rivallan, R., Le Guen, V., Nicolas, M., et al. (2014). De novo transcriptome analysis of Hevea brasiliensis tissues by RNAseq and screening for molecular markers. BMC Genomics 15:236. doi: 10.1186/ 1471-2164-15-236

Shearman, J. R., Sangsrakru, D., Jomchai, N., Ruang-Areerate, P., Sonthirod, C., Naktang, C., et al. (2015). SNP identification from RNA sequencing and linkage map construction of rubber tree for anchoring the draft genome. PLoS One 10:e0121961. doi: 10.1371/journal.pone.0121961

Silva, C. C., Mantello, C. C., Campos, T., Souza, L. M., Gonçalves, P. S., and Souza, A. P. (2014). Leaf-, panel- and latex-expressed sequenced tags from the rubber tree (Hevea brasiliensis) under cold-stressed and suboptimal growing conditions: the development of gene-targeted functional markers for stress response. Mol. Breed. 34, 1035-1053. doi: 10.1007/s11032-0140095-2

Souza, L. M., Gazaffi, R., Mantello, C. C., Silva, C. C., Garcia, D., Le Guen, V., et al. (2013). QTL mapping of growth-related traits in a full-sib family of rubber tree (Hevea brasiliensis) evaluated in a sub-tropical climate. PLoS One 8:e61238. doi: 10.1371/journal.pone.0061238

Tang, C., Yang, M., Fang, Y., Luo, Y., Gao, S., Xiao, X., et al. (2016). The rubber tree genome reveals new insights into rubber production and species adaptation. Nat. Plants 2:16073. doi: 10.1038/nplants.2016.73

Thavamanikumar, S., McManus, L. J., Tibbits, J. F. G., and Bossinger, G. (2011). The significance of single nucleotide polymorphisms (SNPs) in Eucalyptus globulus breeding programs. Aust. For. 74, 23-29. doi: 10.1080/00049158.2011.106 76342

Triwitayakorn, K., Chatkulkawin, P., Kanjanawattanawong, S., Sraphet, S., Yoocha, T., Sangsrakru, D., et al. (2011). Transcriptome sequencing of Hevea brasiliensis for development of microsatellite markers and construction of a genetic linkage map. DNA Res. 18, 471-482. doi: 10.1093/dnares/d sr034

van Berloo, R. (2008). GGT 2.0: versatile software for visualization and analysis of genetic data. J. Hered. 99, 232-236. doi: 10.1093/jhered/esm109

Voorrips, R. E. (2002). MapChart: software for the graphical presentation of linkage maps and QTLs. J. Hered. 93, 77-78. doi: 10.1093/jhered/93.1.77

Weir, B. S. (1979). Inferences about linkage disequilibrium. Biometrics 35, 235-254. doi: $10.2307 / 2529947$

Zhu, C., Gore, M., Buckler, E. S., and Yu, J. (2008). Status and prospects of association mapping in plants. Plant Genome 1, 5-20. doi: 10.3835/ plantgenome2008.02.0089

Conflict of Interest Statement: The authors declare that the research was conducted in the absence of any commercial or financial relationships that could be construed as a potential conflict of interest.

Copyright (c) 2018 de Souza, dos Santos, Rosa, da Silva, Mantello, Conson, Scaloppi, Fialho, de Moraes, Gonçalves, Margarido, Garcia, Le Guen and de Souza. This is an open-access article distributed under the terms of the Creative Commons Attribution License (CC BY). The use, distribution or reproduction in other forums is permitted, provided the original author(s) and the copyright owner(s) are credited and that the original publication in this journal is cited, in accordance with accepted academic practice. No use, distribution or reproduction is permitted which does not comply with these terms. 